\title{
Using Moment of Inertia and Observable Planetary Features to Approximate the Two-Layer Structure of Earth, Jupiter, and Neptune
}

Selena Kimball

University of South Florida

Advisors:

Arcadii Grinshpan, Mathematics and Statistics

Joseph Panzik, Geology, Research Geophysicist

Problem Suggested By: Joseph Panzik

Follow this and additional works at: https://digitalcommons.usf.edu/ujmm

Part of the Mathematics Commons

UJMM is an open access journal, free to authors and readers, and relies on your support:

Donate Now

\section{Recommended Citation}

Kimball, Selena (2019) "Using Moment of Inertia and Observable Planetary Features to Approximate the Two-Layer Structure of Earth, Jupiter, and Neptune," Undergraduate Journal of Mathematical Modeling: One + Two: Vol. 10: Iss. 1, Article 2.

DOI: https://doi.org/10.5038/2326-3652.10.1.4907

Available at: https://digitalcommons.usf.edu/ujmm/vol10/iss1/2 


\title{
Using Moment of Inertia and Observable Planetary Features to Approximate the Two-Layer Structure of Earth, Jupiter, and Neptune
}

\begin{abstract}
This paper examines the interior structure and composition of Earth, Jupiter, and Neptune by using moment of inertia (Mol) and observable planetary features to create approximate two-layer interior structure models. The moment inertia of a uniform sphere, hollow sphere, and a sphere with a shell are derived to calculate the radius and density variables that identify the relationship between the different radii and densities of the two layers. A two-layer model of the planet's interior can then be formulated based on the radius, density, known Mol factor and the surface density or the assumed composition density of the planet. The models created for Jupiter and Neptune are compared to Earth's model and conclude that Jupiter may have a silica-rich core, whereas Neptune's model has inconclusive density and radius results that should be further investigated. Published literature containing current three-layer models of planetary interiors confirm the results produced from the two-layer models in this paper.
\end{abstract}

\section{Keywords}

moment of inertia, two-layer planetary interior model, Earth, Jupiter, Neptune

\section{Creative Commons License}

(c) $($ ) $(9)$

This work is licensed under a Creative Commons Attribution-Noncommercial-Share Alike 4.0 License. 


\section{PROBLEM STATEMENT}

One of the challenges in the field of geophysics is trying to analyze the internal structure and distribution of mass within planetary bodies using information based solely on observations gathered from satellites. Although density is useful in describing the relationship between a planet's mass and the volume of space taken up, it does not describe how the mass is distributed. However, a planet's mass, average radius, and oblateness can be used to calculate the moment of inertia, which can be used to help identify the distribution of mass about a rotating axis.

\section{MOTIVATION}

The moment of inertia (MoI) is a measure of an object's resistance to rotational change, or in this case, being spun upwards or downwards (Nimmo, 2010). Because density is limited by only providing the amount of mass per volume, a planet's moment of inertia can be used to provide more information about the differentiation of a planet's mass. Particularly, the MoI factor can be used to identify if the planet has the internal shape of a uniform sphere, spherical shell, or a sphere and shell. This information can be useful in identifying whether the planet has large structural variations that can represent the composition, such as a dense, rocky core, and the radial proportion of the core to the total size. Additionally, the mass and density distribution can help identify the potential composition of the planetary interiors, which increases our understanding of a planet's chemical composition. Using this information to create conceptual models of planetary composition also provides additional insight into material and mass distribution within our solar system and the processes that led to its formation.

\section{MATHEMATICAL DESCRIPTION AND SOLUTION APPROACH}

Earth has the unique advantage over other planetary bodies in our Solar System of being able to be more rigorously studied through satellite and ground-based observations, such as seismology. Seismology uses the observations of how surface waves and pressure waves traverse through material. The speed of seismic waves is based on the physical properties of the material, such as density, bulk modulus, and shear modulus, which are correlated to the composition and temperature of the material it is transmitted through. Extensive studies of seismic waves and the application of Snell's Law, which determines ray-path trajectory of waves, provides an internal seismic wave-speed and density model of Earth. The model for the internal structure of the Earth is represented by the preliminary reference Earth model (PREM; Dziewonski \& Anderson, 1981). 


\section{Graph 1: Earth's Density vs Radius}

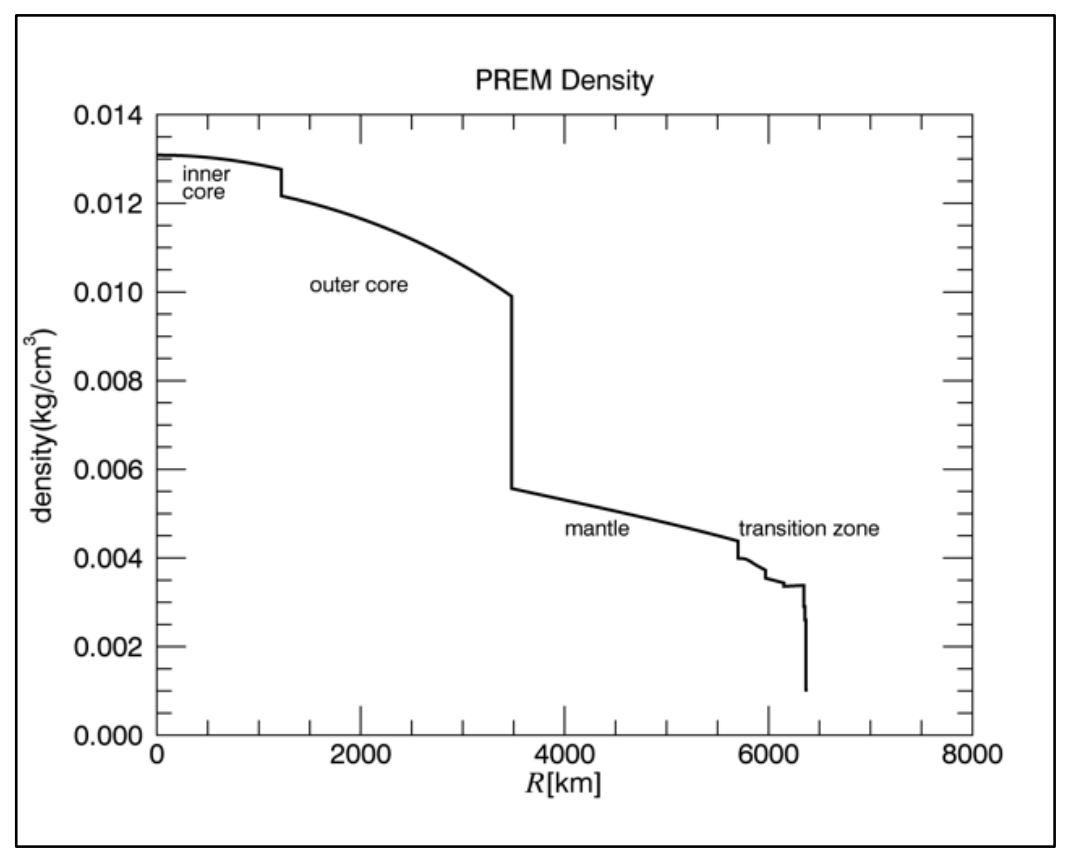

(modified from PREM (Prézeau, 2015))

Graph (1) above shows the model of Earth's internal density structure based on the PREM. Earth's internal density structure has large incremental increases associated with compositional variation, which shows clear distinctions between Earth's mantle, outer core, and inner core. The gradual density transitions are due to increasing pressure and temperature with depth.

Unfortunately, seismic data from other planets is unavailable or sparse due to physical limitations such as extensive surface temperature (Venus), or lack of solid surfaces (Jupiter and Saturn). Instead of using seismic data, the mass distribution can be approximated using observations from satellites to calculate the planet's mass, radius, gravity field and other various orbital parameters. Observational data can also be used to compute the various rotational parameters of a planet, such as spin state, spin axis, and longitudinal displacement to calculate the internal structure (Margot et al., 2012). If a planet has natural satellites, such as moons, then the orbital period and semi-major axis of the orbit can be determined to find the mass (Cook, 1975). Then using the observations described above and Newton's Gravitational Constant (G), the mass can be identified using Kepler's Third Law, where $a$ is the semi-major axis and $P$ is the orbital period: $\quad M=\frac{4 \pi}{G P^{2}} a^{3}$.

With the mass identified, the radius can be calculated by using $\mathrm{G}$, and the gravitational acceleration or the orbital period. Once the mass and radius are calculated, the moment of inertia is used to identify the relationship between the radii and densities of the various layers. However, 
because of the lack of seismic data, we have to assume that other planets are similar to Earth or simplified to only two layers and thus only have two different radii and densities for their internal structure. Furthermore, the mantle density has to be assumed based on the surface density or the known average density of the planet's composition. For example, Jupiter's mantle density is assumed based on the average density of its composition: metallic hydrogen.

As an object is rotating or spinning, it begins to flatten dependent on the speed of rotation, thus planets will have different MoI based on its speed of rotation and how the internal mass is beginning to flatten which is dependent on how the mass is distributed within the planet. Using Earth as a primary model and deriving the moment of inertia of a uniform sphere, hollow sphere, and sphere with a shell, the internal structure of any planet can be determined. A two layer model is used to compare and contrast Earth's interior structure with that of Neptune and Jupiter.

\section{Uniform Sphere}

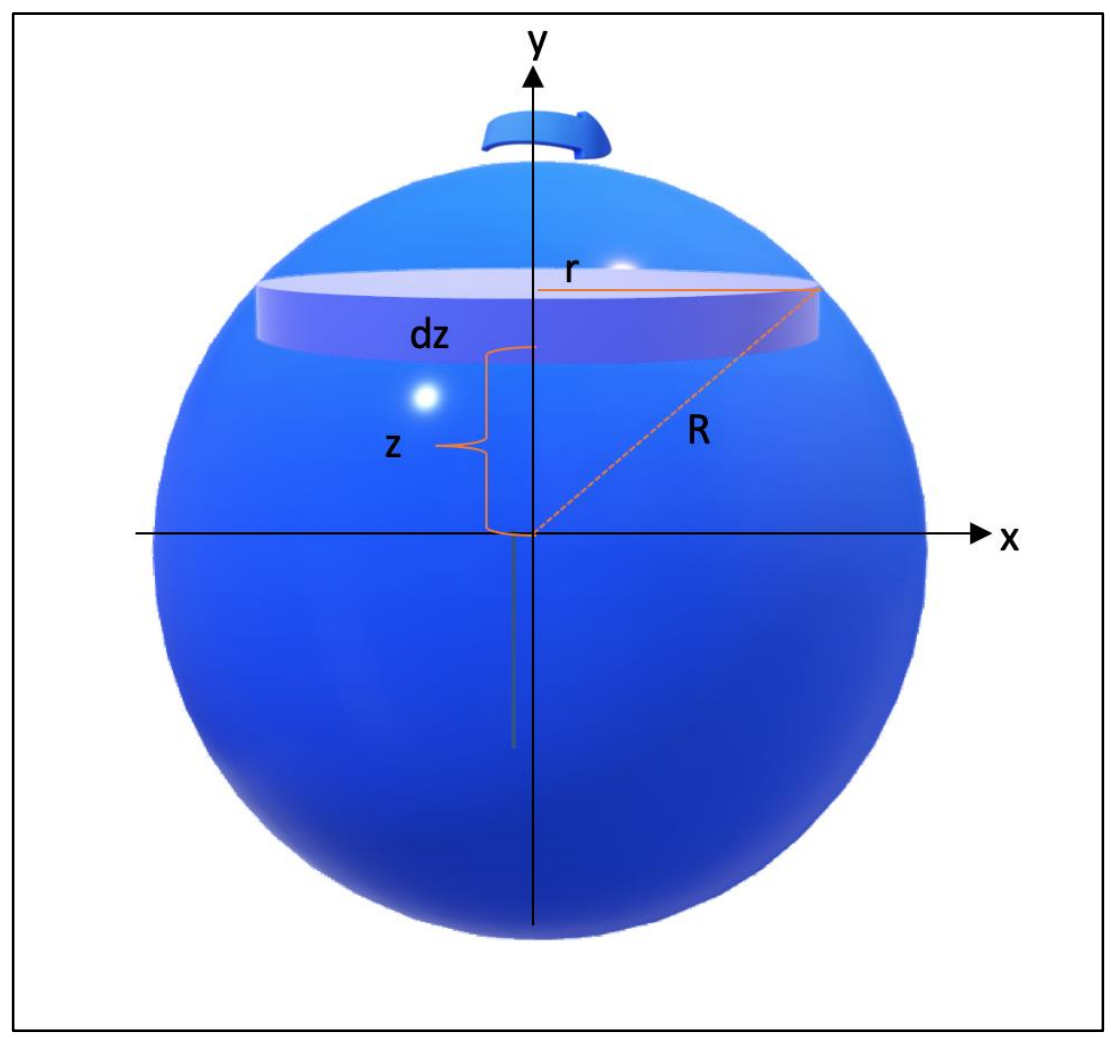

Figure 1. Model of Uniform Sphere 
Figure (1) shows a uniform sphere in both polar and Cartesian coordinates rotating about the $\mathrm{y}$-axis, where $\mathrm{R}$ is the full radius, $\mathrm{z}$ is the distance from the center of the sphere, $\mathrm{dz}$ is the disk thickness, and $\mathrm{r}$ is the disc radius.

The moment of inertia ( $I$ ) of a solid cylinder is shown below, where $r$ is the radius and $d m$ is the differential of the mass $M$, that equals the density $\rho$ multiplied by the volume $V$ :

$$
\begin{array}{r}
I=\frac{1}{2} M R^{2} \Longrightarrow \frac{d I}{d m}=\frac{1}{2} r^{2} \\
d I=\frac{1}{2} r^{2} d m \quad \text { (eq.1) }
\end{array}
$$

The differential $d V=\pi r^{2} d z$ is implemented into the equation with respect to $d z$, the distance of thickness. The simplified equation of MoI becomes:

$$
d I=\frac{1}{2} \rho \pi r^{4} d z \quad(\mathbf{e q . 2 )}
$$

Using the Pythagorean Theorem, $\mathrm{r}^{4}$ can be rewritten in terms of $\mathrm{R}$, the full radius, and $\mathrm{z}$, the distance of thickness from the center of the sphere: $r^{4}=\left(R^{2}-z^{2}\right)^{2}$.

When expanded and added to the equation, the result is: $d I=\frac{1}{2} \pi \rho\left(R^{2}-z^{2}\right)^{2} d z$.

The integral is from - $\mathrm{R}$ to $\mathrm{R}$ because the positive and negative values of the Cartesian coordinate system need to be considered to compute the full sphere. However, since it is an even function, it can be rewritten from 0 to $\mathrm{R}$ :

$$
I=\pi \int_{0}^{R} \rho\left(R^{2}-z^{2}\right)^{2} d z \quad(\mathbf{e q . 3})
$$

After expanding and simplifying, equation (3) can be integrated:

$$
\begin{gathered}
I=\pi \rho \int_{0}^{R}\left(z^{4}-2 R^{2} z^{2}+R^{4}\right) d z=\pi \rho\left(\frac{z^{5}}{5}-\frac{2 R^{2} z^{3}}{3}+R^{4} z\right) \text { from } 0 \text { to } R, \\
=\pi \rho\left(\frac{R^{5}}{5}-\frac{2 R^{5}}{3}+R^{5}\right)=\pi \rho \frac{8 R^{5}}{15} \text { (sol.1) }
\end{gathered}
$$

The equation then has to be converted in terms of density and volume. Since $M=\rho V$, then $d m=$ $\rho d V$. The volume of a sphere and its integration are shown below, with $d m$ equaling the integral of density and volume. The solution for mass is used in later calculations: 


$$
M=\int_{0}^{R} \rho 4 \pi r^{2} d r \Rightarrow M=\rho \frac{4 \pi R^{3}}{3} \quad \text { (eq.4) }
$$

The mass equation above can be rewritten as an expression of density and then substituted into the solution for moment of inertia. The equation for the moment of inertia of a uniform sphere becomes:

$$
I=\left(\rho \frac{4}{3} \pi R^{3}\right)\left(\frac{8 R^{2}}{15}\right)\left(\frac{3}{4}\right)=\frac{2}{5} M R^{2} \text { (sol.2) }
$$

Model of Hollow Sphere. From solution (2), it can be derived that a planet with the internal structure of an uniform sphere has a MoI of 0.40 or close to that value because $\frac{I}{M R^{2}}$ will indicate how much greater the core density is compared to the mantle (Cook, 1975).

\section{Hollow Sphere}

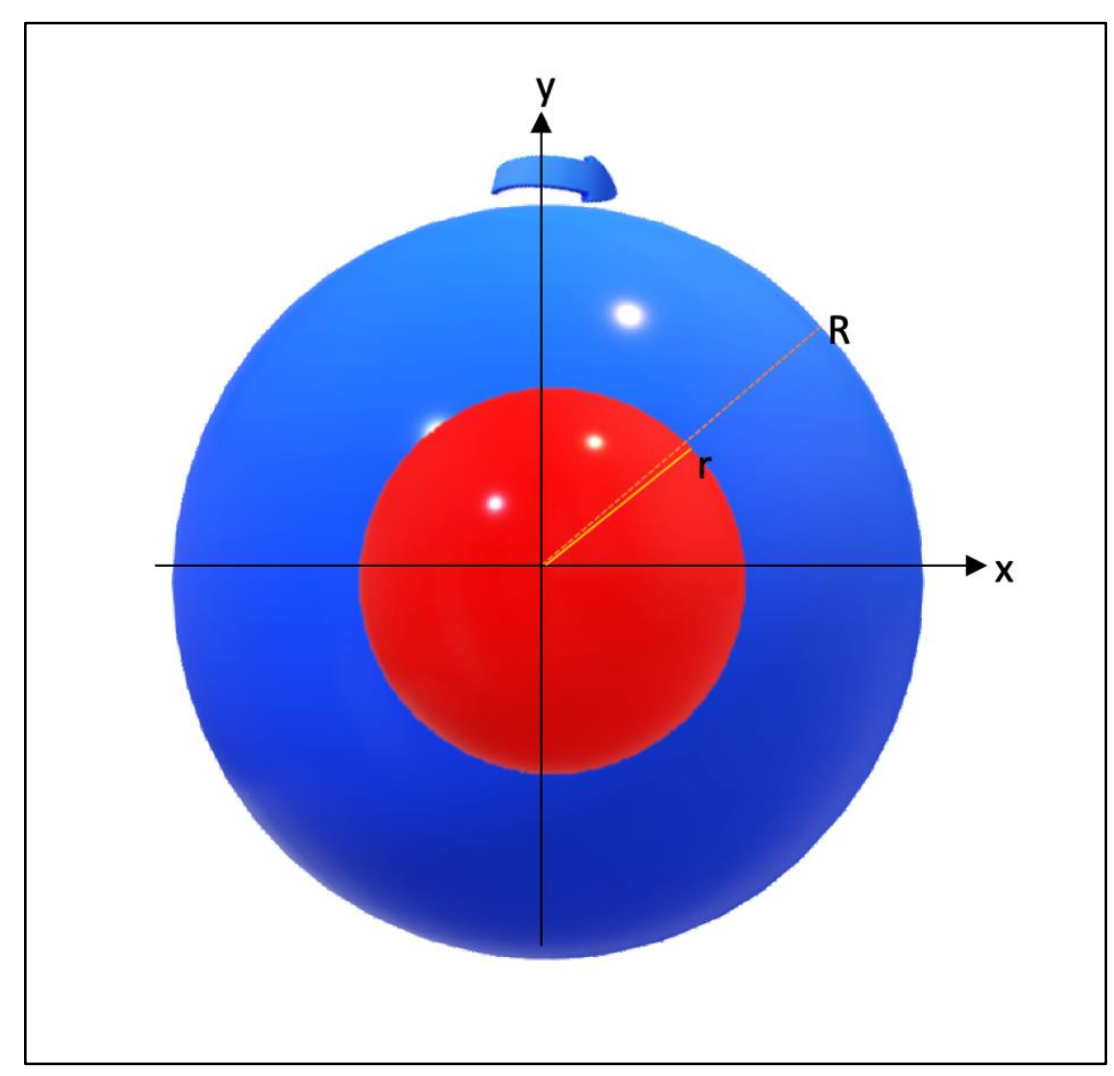

Figure 2. Model of Hollow Sphere 
Figure (2) shows a hollow sphere in both polar and Cartesian coordinate systems rotating about the y-axis, where the red sphere is a hollow area where no mass can exist, $\mathrm{R}$ is the radius of the entire sphere and $\mathrm{r}$ is the radius of the hollow sphere.

To calculate the MoI of a hollow sphere, the following equation has to be used, where the total I is the I of a sphere (sol.1) subtracted from the I of the hollow sphere.

$$
\begin{gathered}
I_{\text {total }}=I_{\text {sphere }}\left\{\begin{array}{l}
R \\
0
\end{array}-I_{\text {sphere }}\left\{\begin{array}{ll}
r \\
0
\end{array} \quad\right. \text { (eq.5) }\right. \\
I_{\text {total }}=\pi \int_{0}^{R} \rho\left(R^{2}-z^{2}\right)^{2} d z-\pi \int_{0}^{r} \rho\left(r^{2}-z^{2}\right)^{2} d z \\
I_{\text {total }}=\pi \rho\left(\frac{8 R^{5}}{15}\right)-\pi \rho\left(\frac{8 r^{5}}{15}\right) \\
I_{\text {total }}=\pi \rho \frac{8}{15}\left(R^{5}-r^{5}\right)
\end{gathered}
$$

The total mass of the hollow sphere can be written as $M_{R}-M_{r}=M$, thus the integral for the mass is from $r$ to $\mathrm{R}$ :

$$
M=\int_{r}^{R} 4 \pi r^{2} \rho d r \Rightarrow M=\frac{4}{3} \pi \rho\left(R^{3}-r^{3}\right)
$$

The equation can then be rearranged to isolate density. Additionally, $r$ can be rewritten to be related to the total radius, $R$, by some constant $a$, which needs to be between 0 and 1 .

$$
\rho=\frac{3}{4 \pi} M\left(\frac{1}{R^{3}-(a R)^{3}}\right) \quad(\text { eq.7) }
$$

The moment of inertia can then be computed by multiplying equations (6) and (7):

$$
\begin{gathered}
I=\pi \frac{8}{15}\left(R^{5}-(a R)^{5}\right) \cdot M \frac{3 \pi}{4}\left(\frac{1}{R^{3}-(a R)^{3}}\right) \\
I=\frac{2}{5} M\left(\frac{R^{5}-(a R)^{5}}{R^{3}-(a R)^{3}}\right)(\mathbf{e q . 8})
\end{gathered}
$$

The indeterminate form can be expanded and L'Hospital's rule or elementary algebra can be applied to find the limit as $a R=r \rightarrow R$. We have

$$
\begin{aligned}
& \frac{R^{5}-(a R)^{5}}{R^{3}-(a R)^{3}}=\frac{(R-a R)\left(R^{4}+R^{3} a R+R^{2}(a R)^{2}+R(a R)^{3}+(a R)^{4}\right)}{(R-a R)\left(R^{2}+R a R+R(a R)^{2}\right)} \text { and } \\
& \qquad \lim _{a R=r \rightarrow R} \frac{R^{5}-(a R)^{5}}{R^{3}-(a R)^{3}}=\lim _{a R=r \rightarrow R} \frac{\left(R^{4}+R^{3} r+R^{2} r^{2}+R r^{3}+r^{4}\right)}{\left(R^{2}+R r+R r^{2}\right)}=\frac{5 R^{2}}{3} .
\end{aligned}
$$


So the MoI can be identified for $a \leq 1$ by simplifying equation (8) such that:

$$
I=\frac{2}{5} M R^{2} \frac{\left(1-a^{5}\right)}{\left(1-a^{3}\right)}, \text { if } a<1, \text { and } I=\frac{2}{3} M R^{2}, \text { if } a=1 \quad(\mathbf{s o l} .3)
$$

\section{Sphere and Shell}

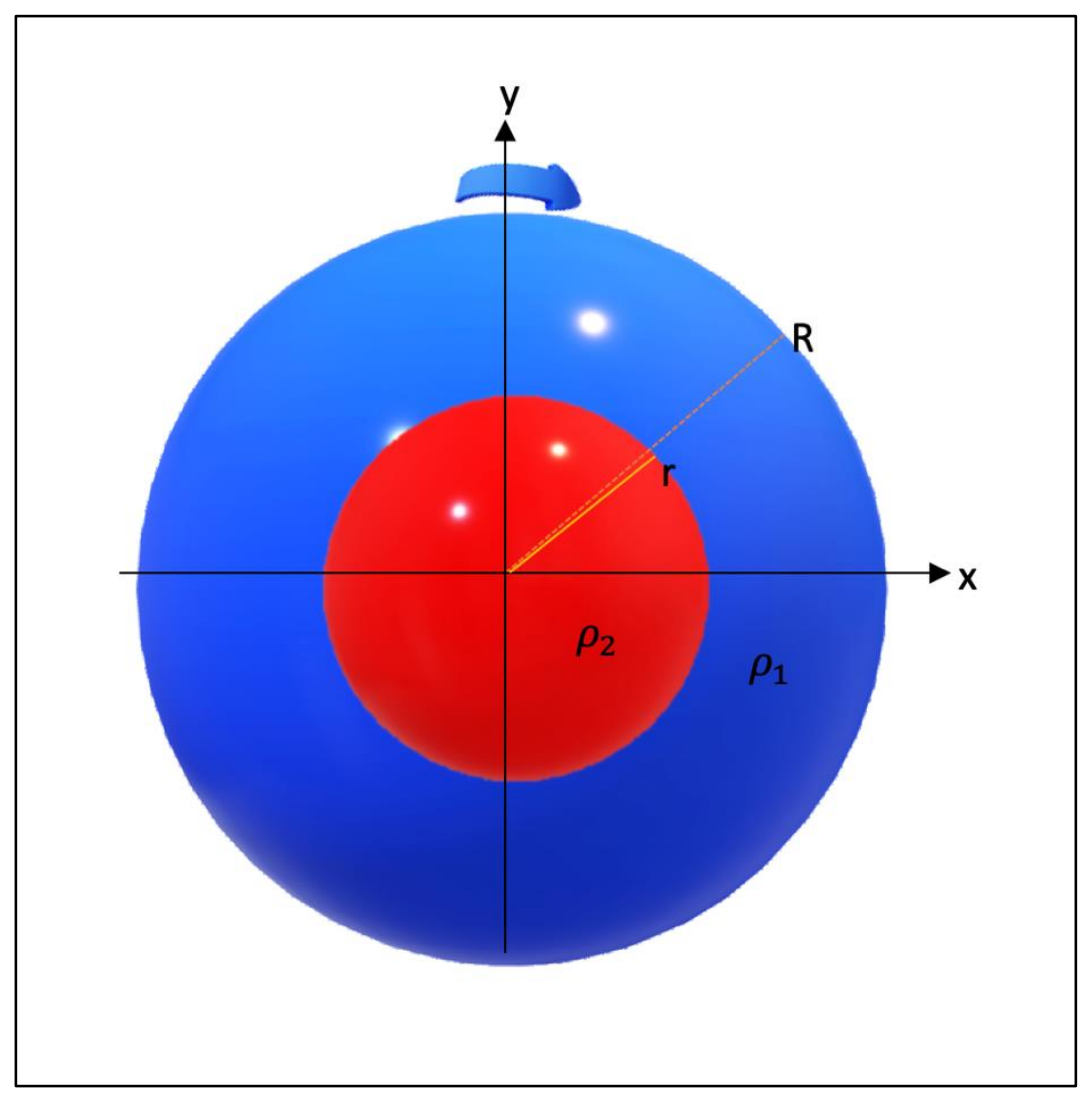

Figure 3. Model of a Sphere and Shell

Figure (3) shows a sphere with a shell of different density and radius. The sphere is in both polar and Cartesian coordinate systems rotating about the y-axis, where the red sphere has density $\rho_{2}$ and radius $r$, and the blue sphere has density $\rho_{1}$ with $R$ radius.

The total moment of inertia for a sphere with a shell is the MoI of a sphere and shell, which are calculated in the previous section, but the shell is now in terms of a second density.

$$
I_{\text {Total }}=I_{\text {sphere }}\left(\left\{\begin{array}{l}
r \\
0
\end{array}, \rho_{1}\right)+I_{\text {sphere }}\left(\left\{\begin{array}{l}
R \\
0
\end{array}, \rho_{2}\right)-I_{\text {shell }}\left(\left\{\begin{array}{l}
r \\
0, \rho_{2}
\end{array}\right)\right.\right.\right.
$$


To calculate the MoI of a sphere with different densities and radii, first the following relationships have to be considered for the total mass, radius, and density of the sphere. The total mass of the sphere is the mass of the smaller sphere plus the mass of the larger sphere:

$$
M=M_{1}+M_{2}
$$

The smaller radius can be presented in terms of $\mathrm{R}: r=a R$, where $a<1$ is some constant. The same relationship can be applied to the density and it can be rewritten with the constant $b$, where $\rho_{2}$ is the density of the core (red sphere) and $\rho_{1}$ is the density of the mantle (blue sphere):

$$
\rho_{2}=b \rho_{1} \text { and } \rho_{1}=\rho
$$

Then the MoI can be integrated with the above considerations and simplified to:

$$
\begin{gathered}
I_{T}=\frac{8 \pi}{15}\left(\rho(a R)^{5}+b \rho\left(R^{5}-(a R)^{5}\right)\right) \\
I_{T}=\frac{8 \pi}{15}\left(a^{5}+b-b a^{5}\right) \rho R^{5}
\end{gathered}
$$

Then the mass of the entire sphere can be calculated as:

$$
\begin{gathered}
M_{T}=\int_{0}^{a R} 4 \pi r^{2} \rho d r+\int_{a R}^{R} 4 \pi r^{2} b \rho d r \\
=\frac{4 \pi}{3}\left(\rho(a R)^{3}+b \rho\left(R^{3}-(a R)^{3}\right)\right.
\end{gathered}
$$

When the above equation is expanded and reduced, it becomes:

$$
M=\frac{4 \pi}{3}\left(a^{3}+b-b a^{3}\right) \rho R^{3}
$$

The solution should then be written in terms of density to plug into equation (9).

$$
\rho=\left(\frac{3 M}{4 \pi}\right)\left(\frac{1}{a^{3}+b-b a^{3}}\right)\left(\frac{1}{R^{3}}\right) \quad \text { (eq.11) }
$$

Equations (9) and (11) are combined to get the moment of inertia for a sphere and shell:

$$
I=\left(\frac{8 \pi}{15}\right)\left(\frac{3 M}{4 \pi}\right)\left(\frac{a^{5}+b-b a^{5}}{a^{3}+b-b a^{3}}\right)\left(\frac{R^{5}}{R^{3}}\right)
$$

$$
\text { When reduced I becomes: } I=\frac{2}{5} M R^{2}\left(\frac{a^{5}+b-b a^{5}}{a^{3}+b-b a^{3}}\right)
$$

To identify the relationship between the radius and density, which provides how the mass is distributed within the planet, the constants $a$ and $b$ have to be solved for. The mass and moment of inertia can be rewritten and replaced with general constants to simplify the 
calculations. For the constant $b$, the relationship between the different densities, the moment of inertia and mass can be used. Then using the solution for $b$, the constant $a$ can be solved for, which represents the relationship between the radii.

For moment of inertia, the constants $a$ and $b$ are isolated by inversing the remaining components and equaling them:

$$
\propto=\left(\frac{5}{2}\right)\left(\frac{I}{M R^{2}}\right)=\frac{b a^{5}+1-a^{5}}{b a^{3}+1-a^{3}}
$$

The constant $a$ can be taken out and then cross multiplied:

$$
\propto=\frac{a^{5}(b-1)+1}{a^{3}(b-1)+1} \Rightarrow \propto a^{3}(b-1)+\propto=a^{5}(b-1)+1
$$

By getting the constant $b$ to one side of the equation, such that:

$$
\begin{gathered}
-a^{5}(b-1)+\propto a^{3}(b-1)=1-\propto \\
\Rightarrow b-1=\frac{1-\propto}{\propto a^{3}-a^{5}}
\end{gathered}
$$

The constant $b$ as it pertains to moment of inertia will equal:

$$
b=\frac{1-\propto}{\propto a^{3}-a^{5}}+1 \quad \text { (sol.5) }
$$

For the mass, the same procedure can be followed to isolate the constant $b$ :

$$
\begin{gathered}
\frac{\beta}{\rho}=\frac{3 M}{4 \pi R^{3} \rho}=b a^{3}+1-a^{3} \\
\frac{\beta}{\rho}=b a^{3}+1-a^{3} \Rightarrow \frac{\beta}{\rho}-1=a^{3}(b-1) \\
\Rightarrow \frac{\beta-\rho}{a^{3} \rho}=b-1
\end{gathered}
$$

The constant $b$ as it pertains to mass is shown below, where $\rho$ is the assumed density of the mantle.

$$
b=1+\frac{\beta-\rho}{a^{3} \rho}
$$

Solutions (5) and (6) can be used to solve for the constant $a$ by equaling them and solving for $a$ :

$$
\frac{1-\propto}{\propto a^{3}-a^{5}}+1=1+\frac{\beta-\rho}{a^{3} \rho}
$$


1 and $\mathrm{a}^{3}$ can be canceled out, simplifying the equation to:

$$
\frac{1-\propto}{\propto-a^{2}}=\frac{\beta-\rho}{\rho}
$$

Both sides of the equation are flipped and $a$ is further isolated:

$$
\frac{\propto-a^{2}}{1-\propto}=\frac{\rho}{\beta-\rho} \Rightarrow \propto-a^{2}=\frac{\rho}{\beta-\rho}(1-\propto)
$$

The sign of $a$ is changed so it is not negative:

$$
\begin{aligned}
& a^{2}-\propto=\frac{\rho}{\beta-\rho}(\propto-1) \\
& a^{2}=\frac{\rho}{\beta-\rho}(\propto-1)+\propto
\end{aligned}
$$

The equation can be further simplified to:

$$
\begin{gathered}
a^{2}=\frac{\propto \rho-\rho}{\beta-\rho}+\propto\left(\frac{\beta-\rho}{\beta-\rho}\right) \Rightarrow \frac{\propto \rho-\rho+\propto \beta-\propto \rho}{\beta-\rho} \\
a^{2}=\frac{\propto \beta-\rho}{\beta-\rho} \quad \text { (sol.7) }
\end{gathered}
$$

Solutions (6) and (7) are used along with the data in table (1) to identify the mass distribution within Earth, Neptune, and Jupiter. For Neptune, the composition density is based on the density of ice, $1000 \mathrm{~kg} / \mathrm{m}^{3}$ and for Jupiter, the composition density is based on metallic hydrogen, $600 \mathrm{~kg} / \mathrm{m}^{3}$ (Nellis, 2001). Using Excel Spreadsheet, the solutions can be inserted to create a two-layer model based on the radii and density of the surface and composition.

Table 1: MoI, Mass, and Radius of Earth, Neptune, and Jupiter

\begin{tabular}{|c|c|c|c|c|}
\hline Planet & MoI & Mass $\left.\mathbf{( 1 0}^{\mathbf{2 4}} \mathbf{k g}\right)$ & Radius (km) & Surface Density $\left(\mathbf{k g} / \mathbf{m}^{\mathbf{3}}\right)$ \\
\hline Earth & 0.3308 & 5.9723 & 6,371 & $-* *$ \\
\hline Neptune & $0.26^{*}$ & 102.413 & 24,622 & 0.45 \\
\hline Jupiter & 0.254 & $1,898.19$ & 69,911 & 0.16 \\
\hline
\end{tabular}

\section{Data collected from Williams (2018)}

*Data from Cook (1975)

**Density of crust and mantle will be used 


\section{DISCUSSION}

The two-layer model for Earth (Appendix, Model 1) created using the known density of the crust, $2850 \mathrm{~kg} / \mathrm{m}^{3}$, is representative of Earth's rocky, silica-rich composition. As the density increases to $4000 \mathrm{~kg} / \mathrm{m}^{3}$, the composition begins to include denser materials like iron metals. The model shows how Earth's mass distribution is concentrated towards the center and dense materials decrease closer to the surface. The two-layer model generated for Jupiter (Appendix, Model 2) based on composition shows a core density similar to that of Earth's crust and mantle. It can be inferred that Jupiter may have similar materials as Earth within its core, such as a core composition of rocky, silica-rich materials. Comparing the models of Earth and Jupiter, provides an approximate core density and composition of Jupiter. Because Neptune has a denser atmosphere than Jupiter, the model depicts a core that is denser than a probable one. The twolayer model for Neptune (Appendix, Model 3) is inadequate because it creates an extremely dense core. It is more likely that the interior composition of Neptune has more complicated layers and more than one material within its core to adjust for the large density.

The surface radius, or the crust of a planet is best represented by using the surface density of the planet for the model, however, the data provide a poor estimate of the radius and density for the core and mantle. Using the composition of the planet's interior can provide a better density distribution based on the core, however, the crust and mantle become obscured in the model. A two-layer model helps to identify the general composition and structure of a planet but it assumes that density does not change with depth and does not provide a detailed representation of the compositional variation dependent on depth. Guillot \& Gautier (2015) published a research paper that created three-layer models of planetary interiors comparing the gas and ice giants. By comparing the two and three-layer models created by Guillot \& Gautier (Appendix, Figures 1-2), the two-layer models can be confirmed and are nearly comparable to the three layer model created for Earth and Jupiter.

\section{CONCLUSION AND RECOMMENDATIONS}

A two-layer model is sufficient for identifying the density and radii distribution of a spherical body that has a moment of inertia. However, due to the lack of seismic data and terrestrial surfaces, as is the case of Neptune, models can still be limited and obscure. As technology advances and additional data are collected, two and three layer models of planetary interiors can be enhanced to further the identification of interior planetary compositions and density distributions. 


\section{NOMENCLATURE}

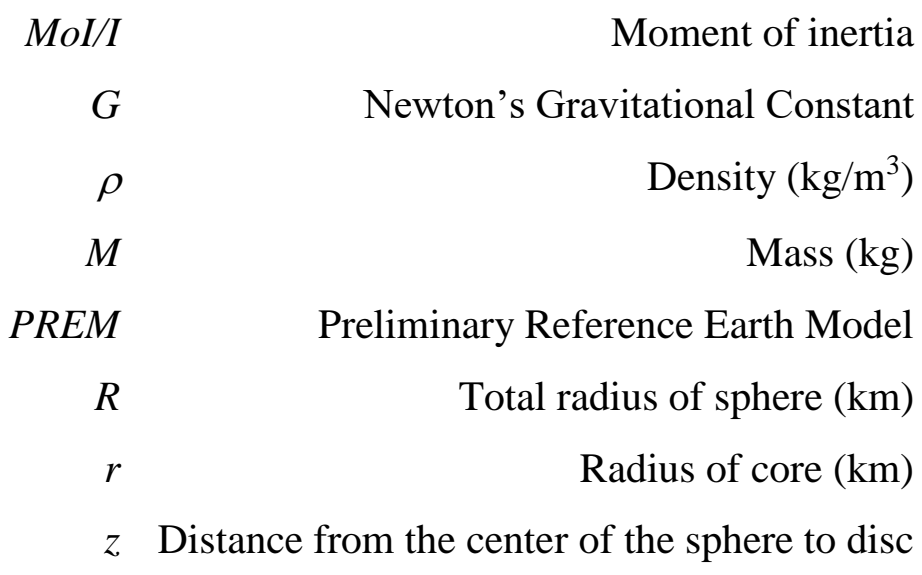

General Constants:

a Total radius as it relates to radius of core

$b \quad$ Total density as it relates to density of core

$\propto$

Moment of inertia (solution 4)

$\beta$

Mass (equation 10) 


\section{REFERENCES}

Cook, A.H (1975, August) The Internal Structures of the Planets, Geophysical Journal International, Volume 42, Issue 2, Pages 285-293.

Dziewonski, A. M., \& Anderson, D. L. (1981). Preliminary reference Earth model. Physics of the Earth and Planetary Interiors, 25, 297-356.

Guillot, T. Gautier, D. (2015) Giant Planets, Treatise on Geophysics, 2nd edition, Elsevier.

Margot, J.-L., Peale, S. J., Solomon, S. C., Hauck, S. A., Ghigo, F. D., Jurgens, R. F., ...Campbell, D. B. (2012). Mercury's Moment of Inertia from Spin and Gravity Data. Journal of Geophysical Research. Planets, 117(E10).

Nellis, W J. (2001, February 6). Metastable Metallic Hydrogen Glass. U.S Department of Energy.

Nimmo, F. (2010). EART162: PLANETARY INTERIORS [PowerPoint lecture]. Retrieved from https://websites.pmc.ucsc.edu/ fnimmo/eart162_10/Week2.pdf

Prézeau, G. (2015). Dense Dark Matter Hairs Spreading Out from Earth, Jupiter and Other Compact Bodies.

Williams, D. R. (2018, July 18). Planetary Fact Sheet- U.S Units. Retrieved from https://nssdc.gsfc.nasa.gov/planetary/factsheet/planet_table_british.html 


\section{APPENDIX}

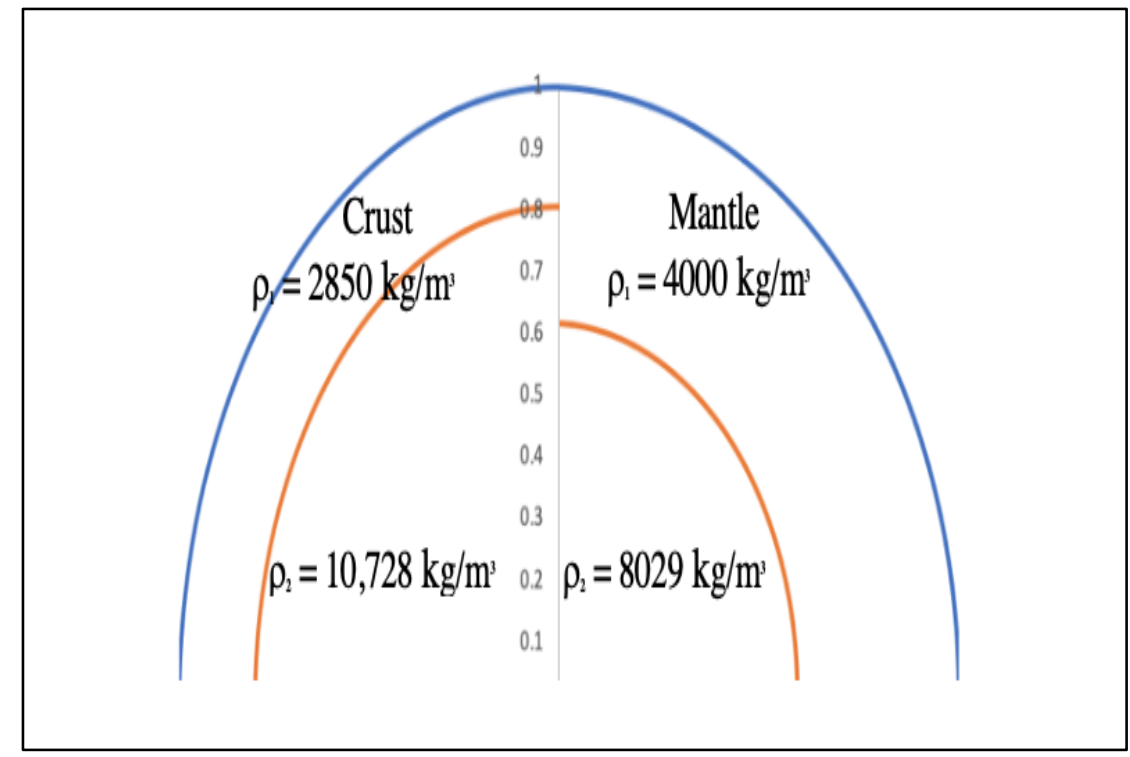

Model 1: Earth's core density and radius based on crust density verses mantle density

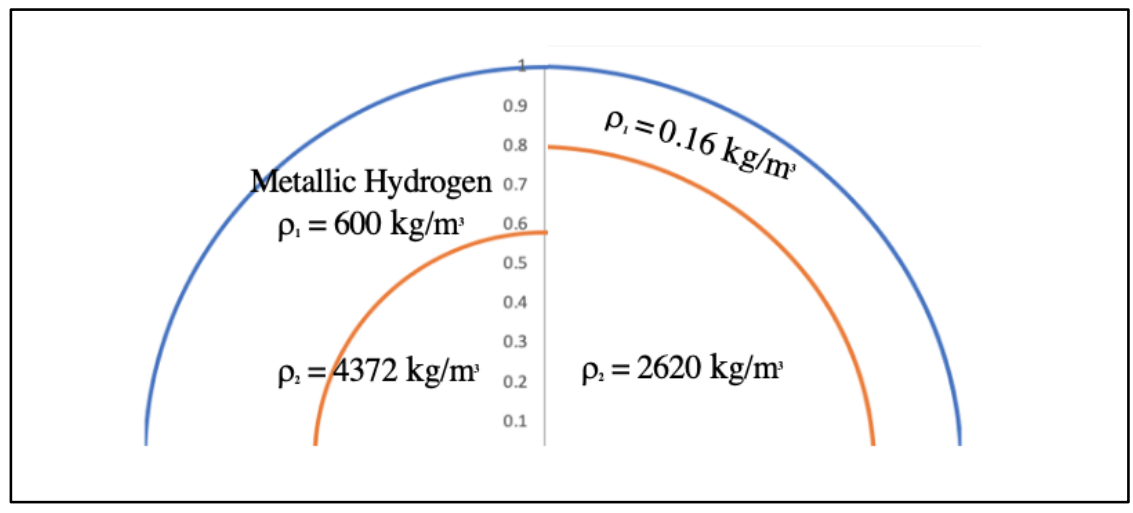

Model 2: Jupiter's core density and radius based on composition verses surface density 


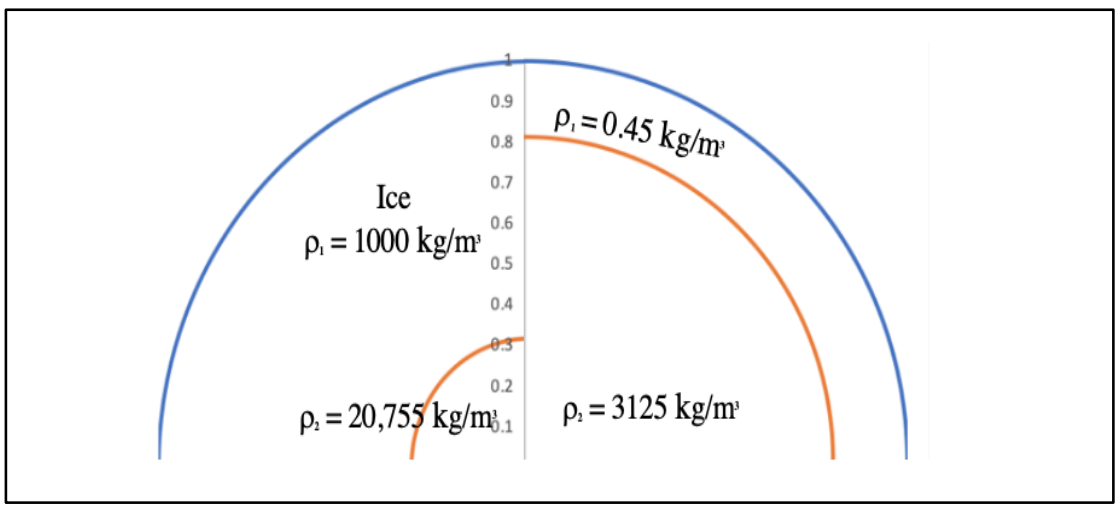

Model 3: Neptune's core density and radius based on composition verses surface density

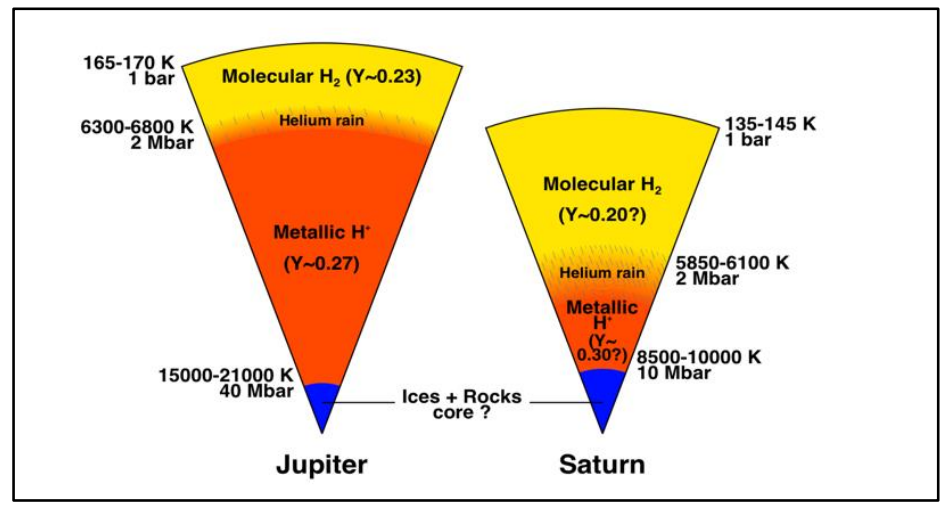

Figure 1: Three-layer model of Jupiter and Saturn, where $Y$ is the mass ratio (Guillot \&Gautier, 2015)

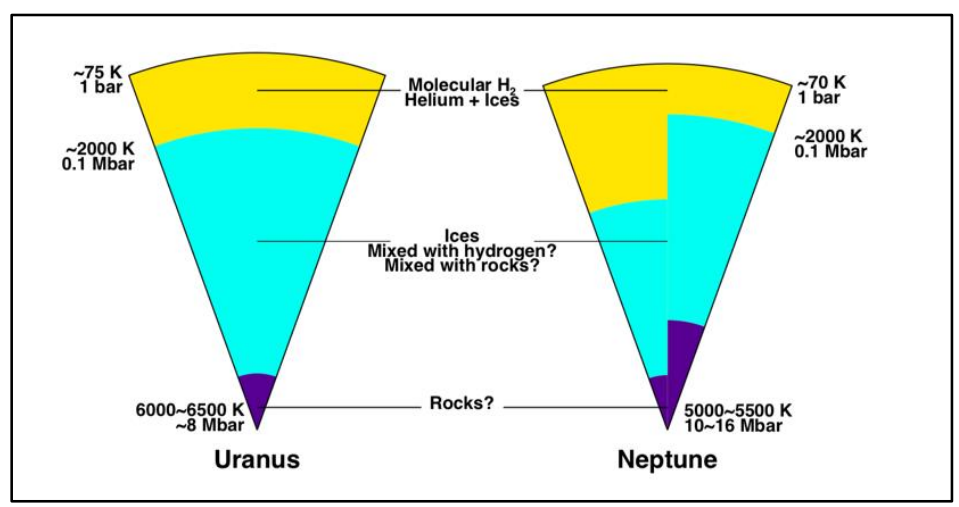

Figure 2: Three-layer model of Neptune and Uranus (Guillot \& Gautier, 2015) 